\title{
NEAR INFRARED COLLOIDAL PbSe/PbS CORE/WING NANOPLATELETS OBTAINED BY A CATION EXCHANGE REACTION
}

\author{
S.A. CHEREVKOV, A.P. LITVIN, T.K. KORMILINA, A.U. DUBAVIK, A.V. SOKOLOVA, \\ A.V. FEDOROV, A.V. BARANOV \\ ITMO University, Center of Information Optical Technologies, Saint Petersburg, Russian Federation, \\ s.cherevkov@itmo.ru
}

https://doi.org/10.37904/nanocon.2019.8526

\begin{abstract}
Two-dimensional colloidal nanocrystals have recently revealed a great potential as optical gain media due to their remarkable optical properties. Besides controlling the size and shape of semiconductor nanocrystals the variation in the chemical composition is another way to control the optical properties of nanomaterials. It is of especial interest to use here the cationic substitution reaction instead of direct synthesis from a Pb precursors, since we may start from cadmium chalcogenide nanocrystals of well controlled size, shape and crystalline structure. So, the cation exchange reaction of cadmium by lead in well-studied colloidal CdSe and core/wing $\mathrm{CdSe} / \mathrm{CdS}$ nanoplatelets has been examined by absorption and photoluminescence spectroscopy.
\end{abstract}

Keywords: Nanoplatelets, core/wing, PbSe, near IR, synthesis

\section{INTRODUCTION}

Semiconductor nanocrystals have been highly attractive materials for research and device applications for decades now. Today much prominence is given to so called nanoplatelets (NPLs), two dimensional colloidal nanocrystals. First acquired in 2006 in the form of nanoribbons [1] they demonstrate electronic structure like that of quantum wells [2]. So that optical properties of NPLs depend chiefly on their thickness, which can be controlled with high precision thanks to modern colloidal synthesis methods [3]. At the same time NPLs have a great advantage over the quantum wells, being free-standing structures, with no dependence on a substrate.

This form is perfect for creating various nanoplatelet-based heterostructures that can inherit NPLs' unique characteristics and vastly improve them. These heterostructures include highly perspective core/shell NPLs, noticed to enhance quantum yield up to $80 \%$ [4]. Studies, conducted in this field demonstrate the synthesis of CdSe NPLs with epitaxially grown CdS and CdZnS shell of controlled thickness [5,6] and later reports regarding core/crown NPLs, where CdSe serves as an exciton funnel that collects the excitons and transfers them to the CdSe core [7].

Their outstanding optical properties favorably distinguish NPLs from other colloidal semiconductor nanocrystals. These nanocrystals are very promising for developing various optoelectronic devices since their two-dimensional geometry is directly compatible with established device designs and processary approaches. It was revealed that high-energy carriers in NPLs relax to the band edge with speeds highly exceeding their recombination velocity. That happens to be the exact quality required for stimulated emission and lasing [8]. Moreover, NPLs show optical gain unreachably high for any other NCs tested for this application. That's why in most recent time it was already possible to develop LEDs [9] including hybrid organic-inorganic ones [10] and semiconductor lasers $[11,12]$ that uses CdSe NPLs and heterostructures based on them as an active emitting layer.

While the CdSe nanoplatelets-based light emitters are undoubtedly a high demand, they can't cover all the possible applications due to the lack of spectral diversity. The chemical substitution of cadmium ions by lead 
in colloidal CdSe nanoplatelets is a simple and promising method for producing 2D nanocrystals which are emitting in the near-infrared region. It is of especial interest to use here the cationic substitution reaction instead of direct synthesis from a $\mathrm{Pb}$ precursors, since we may start from cadmium chalcogenide nanocrystals of well controlled size, shape and crystalline structure. The architectural control of nanosized materials with welldefined shape is important for the success of "bottom-up" approaches toward future nanodevices. However, insight in the physics and prediction of final architecture of nanoscale building blocks are still limited. If we understand the growth mechanism and the shape-guiding process, it will be possible to program the system to achieve nanostructured materials with desired shape and inner crystal morphology.

We suggest that developing a stable and repeatable synthesis procedure of ultrathin $\mathrm{PbSe} / \mathrm{PbS}$ nanoheteroplatelets and researching their optical characteristics and morphology. Devices based on these nanostructures would be extremely useful for solar cells, as well as for integrating them in optical active layers, microfabricated waveguides and lab-on-chip systems [14].

\section{MATERIALS \& METHODS}

\subsection{Chemicals}

Selenium powder (Alfa Aesar, 99.99\%), Oleylamine (Acros, 80-90\%), Oleic acid (Fisher, 70\%), Stearic acid (Fisher, $>97 \%$ ), Cadmium acetate dihydrate (Sigma-Aldrich, >98\%), 2-ethylhexanoic acid (Sigma-Aldrich, 99\%), 1-Octadacene (Sigma-Aldrich, $>95 \%$ ), Hexane (Sigma-Aldrich, $>97 \%$ ), lead iodide (Sigma-Aldrich, 99\%), Cadmium oxide (Sigma-Aldrich, 99.5\%), Myristic acid (Sigma-Aldrich, $\geq 98.0 \%$ ), Tetrachloroethylene (Sigma-Aldrich, anhydrous, $\geq 99 \%$ ), Isopropanol and Methanol (Vekton, puriss). All chemicals were used as received without further purification, except oleylamine which was centrifuged before use.

\subsection{Nanoplatelets Synthesis}

Synthesis of CdSe nanoplatelets. CdSe and CdSe-CdS nanoheteroplatelets nanoplatelets were synthesized by procedure [13]. In a three-neck reaction flask, cadmium oxide $(0.3 \mathrm{mmol})$, myristic acid $(0.72 \mathrm{mmol})$ and ODE $(2 \mathrm{ml})$ were heated to complete dissolution of Cd oxide. Next, ODE $(13 \mathrm{ml})$ was added and the reaction mixture degassed at $100{ }^{\circ} \mathrm{C}$ in vacuum. After cooling to room temperature, selenium powder $(0.12 \mathrm{mmol})$ was added to the reaction flask and the flask purged with argon. The reaction mixture was heated quickly to $190^{\circ} \mathrm{C}$ under argon flow and intense stirring and cadmium acetate $(0.25 \mathrm{mmol})$ added rapidly to the reaction mixture. The mixture was heated in 5 minutes to $240{ }^{\circ} \mathrm{C}$ and stirred at this temperature to grow the nanoplatelets of desired size. After the completion of the process the reaction mixture was cooled down rapidly to $150{ }^{\circ} \mathrm{C}$ and $1 \mathrm{ml}$ of oleic acid added to increase the colloidal stability of nanoplatelets. Then, the reaction mixture was cooled down to $80^{\circ} \mathrm{C}$ and the nanocrystals were deposited with isopropanol, centrifuged, washed several times with isopropanol and redispersed in octadecene.

Synthesis of CdSe-CdS nanoheteroplatelets. Purified CdSe nanoplatelets, cadmium acetate $(0.5 \mathrm{mmol})$, oleic acid $(0.08 \mathrm{mmol})$ and ODE $(15 \mathrm{ml})$ were introduced into a three-neck flask. The reaction mixture was degassed under vacuum at room temperature for $40 \mathrm{~min}$ and heated to $200{ }^{\circ} \mathrm{C}$ under argon and slow stirring. At this temperature the solution of $\mathrm{Cd}$ and $\mathrm{S}$ precursors in ODE was added dropwise into the reaction mixture. To prepare the precursor solution $0.3 \mathrm{mmol}$ of sulfur was dissolved in $3 \mathrm{ml}$ of ODE and $0.42 \mathrm{mmol}$ of CdO dissolved in $2 \mathrm{ml}$ of ODE with $1.2 \mathrm{mmol}$ of 2-ethylhexanoic acid. Then, both solutions were combined and purged with argon. The heteroplatelets were left to grow during $10 \mathrm{~min}$ and after the flask was cooled down.

Cation exchange. $\mathrm{PbSe} / \mathrm{PbS}$ nanoheteroplatelets were synthesized by modified procedures [14]. In a typical synthesis, a mixture of Pbl2 $(0.065 \mathrm{mmol}), 1 \mathrm{ml}$ OIAm, and $4 \mathrm{ml} \mathrm{ODE}$ was degassed at $100{ }^{\circ} \mathrm{C}$ for 30 minutes. The temperature was lowered to $80^{\circ} \mathrm{C}$ and the flask was filled with nitrogen. At this temperature, $\mathrm{CdSe} / \mathrm{CdS}$ NPLs in toluene were swiftly injected. In order to prepare this injection solution, typically, a $300 \mathrm{mkl}$ portion of 
a concetrated CdSe-CdS nanoheteroplatelets dispersion in $2 \mathrm{ml}$ toluene. After 3 hours of the cation exchange the NPLs were precipitated in centrifuge tubes containing toluene $(1.5 \mathrm{ml})$, isopropanol $(4 \mathrm{~mL})$ and oleic acid $(0.2 \mathrm{~mL})$. The exchanged NPLs were dispersed in TCE.

\subsection{Electron Microscope Imaging}

For conventional TEM observations, the samples were prepared by drop-casting concentrated NPLs solutions onto carbon-coated 200 mesh copper grids, and the measurements were performed with a scanning electron microscope Merlin (Zeiss) at $30 \mathrm{kV}$ accelerating voltage.

\subsection{Absorbance and PL spectroscopy}

We studied electronic energy structure of by means of absorbance (Abs) and photoluminescence (PL) spectroscopy. All measurements were performed at room temperature. Abs measurements were carried out with a UV-Probe 3600 (Shimadzu) spectrophotometer and the PL signal passed through the Acton SP2150i monochromator and was recorded using an avalanche InGaAs/lnP single-photon avalanche diode (Micro Photon Devices).

\section{RESULTS AND DISCUSSION}

At the initial stage, CdSe nanoplatelets of 5 monolayers with characteristic for such structures luminescence and absorption bands were obtained (Figure 1). Electron microscopic images of the obtained samples show, that we have indeed acquired square NPLs with lateral size of approximately $20 \mathrm{~nm}$. Hence suggested synthesis procedure allows to obtain NPLs of desired thickness that are monodisperse by their lateral sizes. Some of NPLs in the images are standing on their edge and self-assembly of NPLs in distinguishing stack structures can be observed.

a)

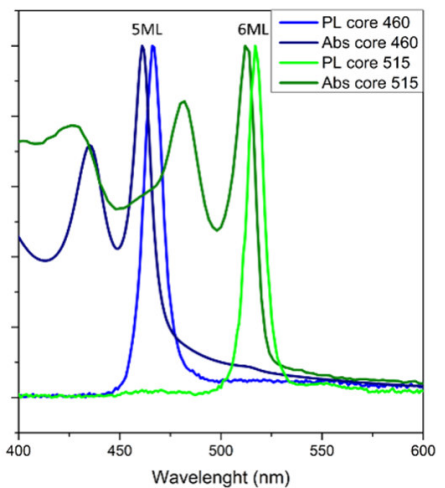

b) $20 \mathrm{~nm}$

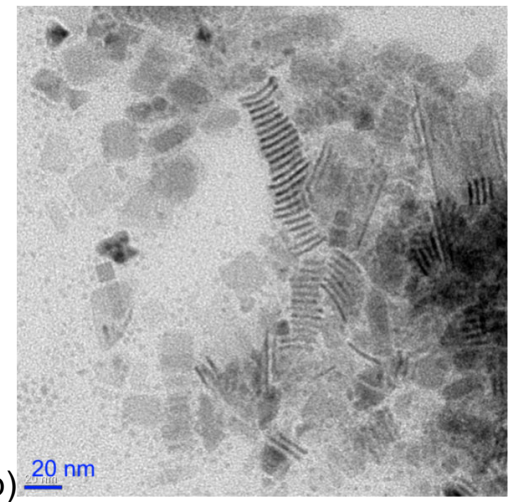

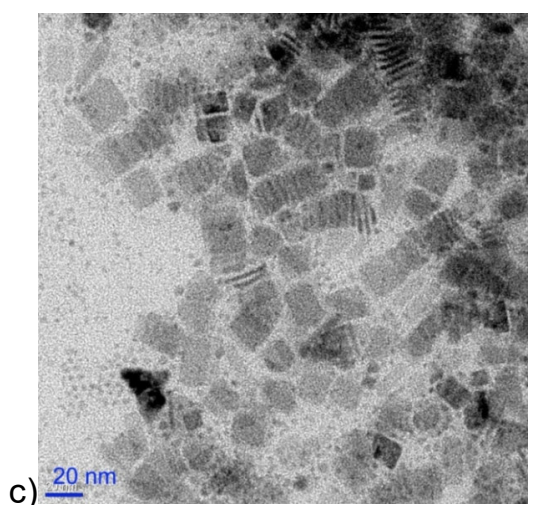

Figure 1 a) PL and Abs spectra of NPLs and TEM images of b) $5 \mathrm{ML}$ and c) $6 \mathrm{ML}$ thick NPLs

Following, the procedure of lateral extension by the CdS semiconductor was carried out, i.e., the so-called core/wing heterostructures were obtained (Figure 2). The absorption spectrum of nano-heteroplatelets contains new absorption bands that correspond to CdS nanoplatelets with a same thickness of 5 monolayers. The luminescence spectrum of $\mathrm{CdSe} / \mathrm{CdS}$ nano-heteroplatelets is practically unchanged compared the luminescence spectrum of the initial NPLs with the exception of a small shift of $5 \mathrm{~nm}$ to the long-wavelength region. However, there is no maximum on the luminescence spectrum that would correspond to these new optical transitions in absorption. This behavior indicates the formation of a heterostructure with efficient charge or energy transfer. The quantum yield of nanoplates after the growth of the wing increased from 11 to $24 \%$. Electron microscopic images of the NPLs after the lateral extension procedure show that the nanocrystals are rectangular with an irregular boundary with a maximum length of $150-200 \mathrm{~nm}$. 


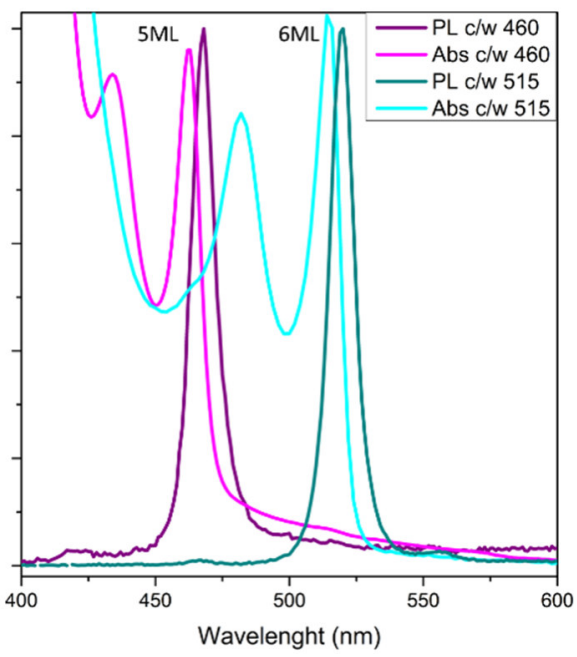

a)

Figur

Wavelenght $(\mathrm{nm})$

Figure 2 a) PL and Abs spectra of core/wings CdSe/CdS NPLs and b) TEM image of 6 ML thick NPLs

After the procedures of substitution, the luminescence and absorption bands of structures move to the near IR region of $1200-1300 \mathrm{~nm}$, which indicates the complete replacement of cadmium atoms by lead in the composition of CdSe / CdS nano-heteroplatelets. SEM images of our nanocrystals show that we acquired nanoplates and their lateral size is approximately $200 \mathrm{~nm}$ (Figure 3). Therefore, the proposed cationic substitution procedure allows one to preserve the two-dimensional shape of nanocrystals.

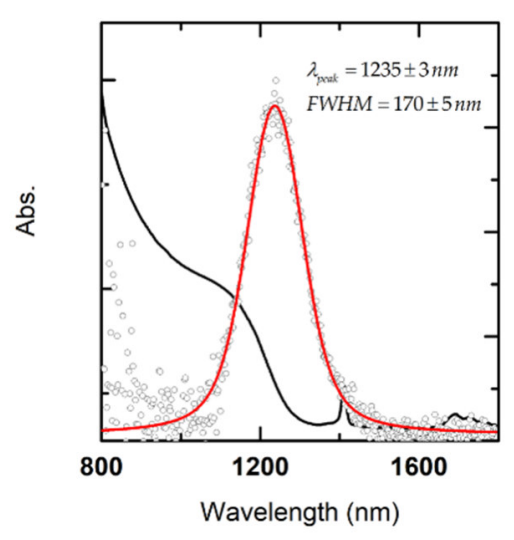

a)

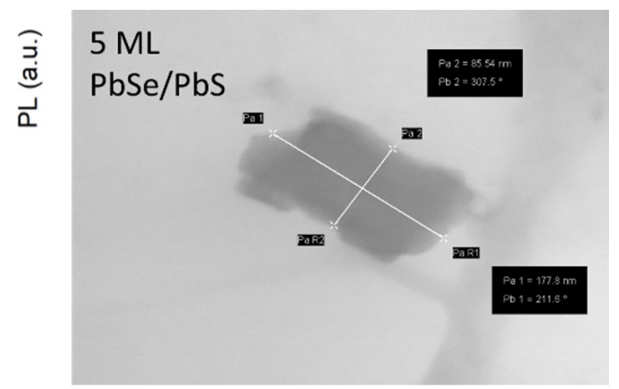

b)

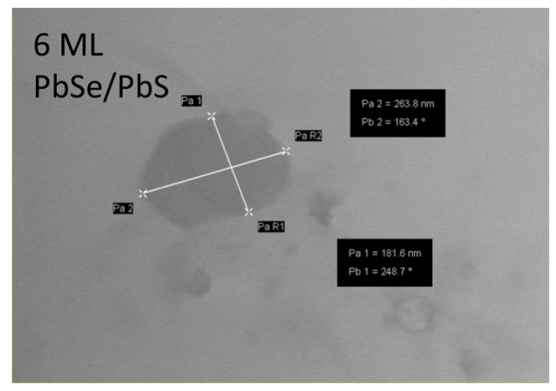

c)

Figure 3 a) PL and Abs spectra of $5 \mathrm{ML} \mathrm{PbSe/PbS} \mathrm{NPLs} \mathrm{and} \mathrm{SEM} \mathrm{images} \mathrm{of} \mathrm{b)} 5 \mathrm{ML}$ and c) $6 \mathrm{ML}$ thick IR NPLs

\section{CONCLUSION}

Present work contains our study on optical properties and morphology of two-dimensional core/wings $\mathrm{PbSe} / \mathrm{PbS}$ nanostructures. We have developed a reproducible synthesis procedure for obtaining ultrathin NPLs with the near infra-red emission.

We have studied absorbance and photoluminescence characteristics of these NPLs. The obtained results fully comply with the literature data for this class of nanoobjects. They demonstrate several fundamental differences from cadmium compounds NPLs due to the difference in the energy structures of nanocrystals having a zinc blende lattice in the case of cadmium and a cubic salt lattice in the case of lead. The absorption spectrum shows a clearly distinguishable step, and not narrow exciton maxima, like cadmium compounds NPLs. This 
can be partially explained by the dispersion of the thickness of the nanoplates in the sample, as well as the large value of the dielectric constant of lead salts.

Lead-based NPLs are characterized by a large surface area up to several microns, which together with luminescent parameters makes them potentially applicable in photovoltaics.

\section{ACKNOWLEDGEMENTS}

\section{This work was supported by the Russian Science Foundation (Agreement 19-13-00332).}

\section{REFERENCES}

[1] JOO, J., SON, J. S., KWON, S. G., YU, J. H., \& HYEON, T. Low-temperature solution-phase synthesis of quantum well structured CdSe nanoribbons. Journal of the American Chemical Society, 2006, vol.128, no.17, pp. 5632-5633.

[2] ITHURRIA, S., DUBERTRET, B. Quasi 2D colloidal CdSe platelets with thicknesses controlled at the atomic level. Journal of the American Chemical Society, 2008, vol. 130, no. 49, pp. 16504-16505.

[3] ITHURRIA S., BOUSQUET G., DUBERTRET B. Continuous transition from 3D to 1D confinement observed during the formation of CdSe nanoplatelets. Journal of the American Chemical Society, 2011, vol. 133, no. 9, pp. 3070-3077.

[4] LI, Z., PENG, X. Size/shape-controlled synthesis of colloidal CdSe quantum disks: Ligand and temperature effects. Journal of the American Chemical Society, 2011, vol. 133, no.17, pp. 6578-6586.

[5] TESSIER, M. D., MAHLER, B., NADAL, B., HEUCLIN, H., PEDETTI, S., \& DUBERTRET, B. Spectroscopy of colloidal semiconductor core/shell nanoplatelets with high quantum yield. Nano letters, 2013, vol. 13, no.7, pp. 3321-3328.

[6] MAHLER, B., NADAL, B., BOUET, C., PATRIARCHE, G., \& DUBERTRET, B. Core/Shell colloidal semiconductor nanoplatelets. Journal of the American Chemical Society, 2012, vol. 134, no.45, pp. 18591-18598.

[7] TESSIER, M. D., SPINICELLI, P., DUPONT, D., PATRIARCHE, G., ITHURRIA, S., \& DUBERTRET, B. Efficient exciton concentrators built from colloidal core/crown CdSe/CdS semiconductor nanoplatelets. Nano letters, 2013, vol. 14, no. 1, pp. 207-213.

[8] PELTON, M., ITHURRIA, S., SCHALLER, R. D., DOLZHNIKOV, D. S., \& TALAPIN, D. V. Carrier cooling in colloidal quantum wells. Nano letters, 2012, vol. 12, no.12, pp. 6158-6163.

[9] CHEN, Z., NADAL, B., MAHLER, B., AUBIN, H., \& DUBERTRET, B. Quasi-2D Colloidal Semiconductor Nanoplatelets for Narrow Electroluminescence. Advanced Functional Materials, 2014, vol. 24, no. 3, pp. 295-302.

[10] VITUKHNOVSKY, A. G., LEBEDEV, V. S., SELYUKOV, A. S., VASHCHENKO, A. A., VASILIEV, R. B., \& SOKOLIKOVA, M. S. Electroluminescence from colloidal semiconductor CdSe nanoplatelets in hybrid organicinorganic light emitting diode. Chemical Physics Letters, 2015, vol. 619, pp. 185-188.

[11] SHE, C., FEDIN, I., DOLZHNIKOV, D. S., DEMORTIËRE, A., SCHALLER, R. D., PELTON, M., \& TALAPIN, D. V. Low-threshold stimulated emission using colloidal quantum wells. Nano letters, 2014, vol.14, no. 5, pp. 2772-2777.

[12] GUZELTURK, B., KELESTEMUR, Y., OLUTAS, M., DELIKANLI, S., \& DEMIR, H. V. Amplified spontaneous emission and lasing in colloidal nanoplatelets. ACS nano, 2014, vol. 8, no. 7, pp. 6599-6605.

[13] PRUDNICAU, A., CHUVILIN, A., ARTEMYEV, M. (2013). CdSe-CdS Nanoheteroplatelets with Efficient Photoexcitation of Central CdSe Region through Epitaxially Grown CdS Wings. Journal of the American Chemical Society, 2013, vol. 135 no. 39, pp. 14476-14479.

[14] GALLE, T., SAMADI KHOSHKHOO, M., MARTIN-GARCIA, B., MEERBACH, C., SAYEVICH, V., KOITZSCH, A., EYCHMULLER, A. Colloidal PbSe Nanoplatelets of Varied Thickness with Tunable Optical Properties. Chemistry of Materials, 2019. 